\title{
A SLIDING MODE CONTROL FOR FOUR-WIRE SHUNT ACTIVE FILTER
}

\author{
Farid HAMOUDI* - Aziz CHAGHI* \\ Mouloud ADLI ${ }^{* *}$ - Hocine AMIMEUR ${ }^{*}$
}

\begin{abstract}
The present paper deals with the sliding mode control of a three-phase four-wire shunt active filter SAF, to improve phase-current waveform, neutral current mitigation and reactive power compensation in electric power distribution system. The sliding mode is formulated using elementary differential geometry, then the control vector is deduced from the sliding surface accessibility using the Lyapunov stability. The algorithm used to establish the current references for the sliding mode controller is based on the instantaneous real and imaginary power theory for four-wire system. It will be seen that this method permits to synthesis the control vector with simple manner, and finally, the obtained simulation results confirm that the above objectives are satisfied.
\end{abstract}

K e y w o r d s: sliding mode control, four-wire active filter, harmonics current compensation

\section{INTRODUCTION}

The increased severity of harmonic pollution in power distribution network has attracted the attention to develop dynamic and adjustable solutions to the power quality problems giving rise to active filter [1-5]. Threewire active filtering provides compensation of harmonics, reactive power, but can't compensates zero sequence components caused by single-phase non-linear loads inherently generate more harmonics than three-phase nonlinear loads, for this reason, four-wire active filtering is recommended in distribution system [3-5].

After synthesizing the reference currents, the voltage source inverter must inject these components in the point of common coupling with minimum error and fast response, this objective requires an appropriate current control method, in this regard, two different approaches are generally adapted; the fixed frequency control and the variable structure control (VSC). The first one requires a linear approach of the system to synthesize the control law. This approach can be advantageous for the fixed frequency but it is not very adapted to multi-frequencies signals. In fact the proportional integral controller performed traditionally for this approach are known for there handicap to regulate correctly alternating references.

The sliding mode control (SMC) which is derived from the theory of variable structure control introduced since long time [6], is a known discontinuous control technique which takes in account the time varying topology of the controlled system. Thus this technique is naturally suitable to control systems based on power electronics devices in general [7-9], and active filter as particular case of these systems $[10,11]$. It is characterized by simplicity implementation, high robustness in presence of uncertainly in system parameters, and fast response. For these reasons, the sliding mode control can be successfully applied to achieve harmonics references regulation.
The purpose of this paper is the application of the sliding mode control to a three-phase four-wire shunt active filter based on three-leg voltage source inverter (VSI).

The layout of this paper is as follows: The system description and the establishment of the differential equations of the system will make the object of the first part of this paper. After that, we will explain briefly the algorithm of the reference current computation based on the known $p q$-theory. The sliding mode control to active filter current control is then described in detail, and finally simulation results considering load variation will be given to measure the performances and the validity of the proposed control.

\section{SYSTEM DESCRIPTION AND MODELING}

Figure 1 illustrates the configuration of the four-wire shunt active filter under study in this paper. The power circuit is based on three-phase three-leg voltage source inverter, connected to the network through a first order passive filter $\left(L_{C}, r_{C}\right)$. At the DC side the bus is constituted of two capacitors $C_{1}=C_{2}=C$ with a midpoint connected to the neutral wire of the network. The objective of this SAF topology is to inject a current vector $i_{c a b c N}$ in the mains in order to compensate for harmonics, reactive and neutral components of the load currents, keeping the DC-link voltages $V_{C 1}$ and $V_{C 2}$ balanced and in an admissible range. To establish the dynamic equations of the system, let suppose that the power switches $S_{k}$ can be assumed ideals, then the output voltage for each phase $k$ to neutral can be expressed as follows

$$
v_{c k}=d_{k} V_{C 1}-\bar{d}_{k} V_{C 2} \text {. }
$$

Where the switch position functions $d_{k}$ define the states of the power switches $S_{k}$ and $S_{k}^{\prime}$ as follows

$$
d_{k}= \begin{cases}1 & \text { if } S_{k} \text { on, } S_{k}^{\prime} \text { off, } \\ 0 & \text { if } S_{k} \text { off, } S_{k}^{\prime} \text { on }\end{cases}
$$

\footnotetext{
* Department of Electrical Engineering, Batna University, Street of Med El Hadi Boukhlouf, 05000 Batna, Algeria, ${ }^{* *}$ Department of Electrical Engineering, Bejaia University, Street of Targa Ouzemour, 06000 Bejaia, Algeria, f_hamoudi@yahoo.fr
} 


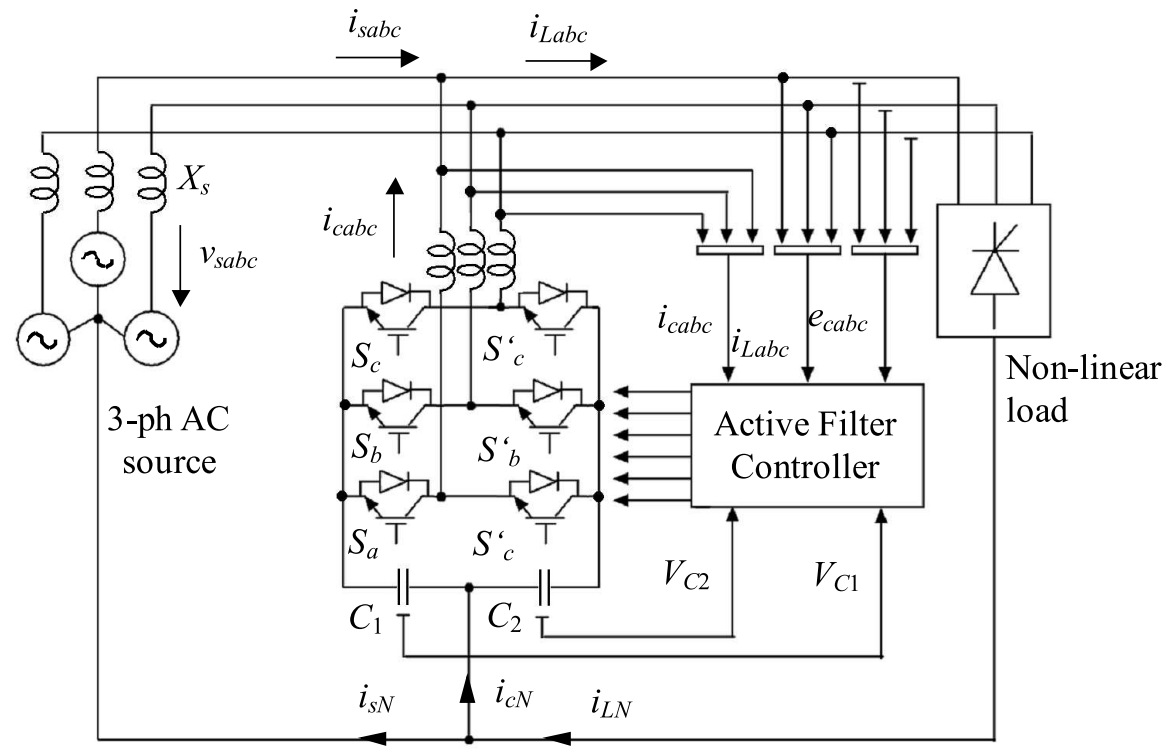

Fig. 1. The configuration of the four-wire shunt active filter under study

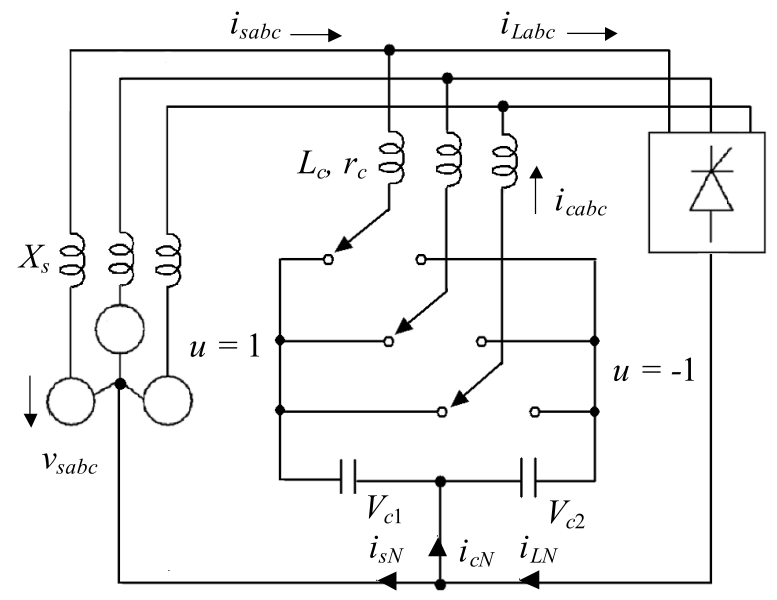

Fig. 2. Equivalent circuit of the configuration from Fig.1

$V_{C 1}$ and $V_{C 2}$ are the voltage at the two capacitors of the DC-bus, related to $d_{k}$ and the active filter currents $i_{c k}$ as follows

$$
\begin{aligned}
& C \frac{\mathrm{d} V_{C 1}}{\mathrm{~d} t}=\sum_{k=a, b, c} d_{k} i_{c k}, \\
& C \frac{\mathrm{d} V_{C 2}}{\mathrm{~d} t}=\sum_{k=a, b, c}-\bar{d}_{k} i_{c k} .
\end{aligned}
$$

Note that $d_{k}=\bar{d}_{k}-1$.

The interaction between the voltage source inverter is scribed by the following differential equation

$$
L_{c} \frac{\mathrm{d} i_{c k}}{\mathrm{~d} t}=-r_{c} i_{c k}+v_{c k}-e_{k} .
$$

Where $e_{k}$ represent the mains voltages at the point of common coupling.

Replacing the function $d_{k}$ with a new function $u_{k}$ defined as follows

$$
u_{k}=2 d_{k}-1
$$

Thus

$$
u_{k}= \begin{cases}1 & \text { when } d_{k}=1 \\ -1 & \text { when } d_{k}=0\end{cases}
$$

With this new function, the power circuit in Fig. 1 can be replaced by an equivalent circuit as shown in Fig. 2, and then the equation (1) is rewritten as follows

$$
u_{c k}=\frac{1}{2} u_{c k}\left(V_{C 1}+V_{C 2}\right)+\frac{1}{2}\left(V_{C 1}-V_{C 2}\right) .
$$

Putting $V_{C 1}+V_{C 2}=V_{d c}$, and $V_{C 1}-V_{C 2}=\Delta V_{d c}$ which represent respectively the DC-bus voltage and the unbalance between the two capacitors. Replacing, (2), (3) and (4) can be rewritten respectively as follows

$$
\begin{aligned}
\frac{\mathrm{d} V_{C 1}}{\mathrm{~d} t} & =\frac{1}{2 C}\left(\sum_{k=a, b, c} u_{k} i_{c k}+\sum_{k=a, b, c} i_{k}\right), \\
\frac{\mathrm{d} V_{C 2}}{\mathrm{~d} t} & =\frac{1}{2 C}\left(\sum_{k=a, b, c} u_{k} i_{c k}-\sum_{k=a, b, c} i_{k}\right), \\
L_{c} \frac{\mathrm{d} i_{c k}}{\mathrm{~d} t} & =-r_{c} i_{c k}+\frac{1}{2} u_{k} V_{d c}+\frac{1}{2}\left(V_{C 1}-V_{C 2}\right)-e_{k} .
\end{aligned}
$$

Finally, these equations are rearranged under matrix from

$$
\begin{aligned}
\frac{\mathrm{d}}{\mathrm{d} t}\left[\begin{array}{c}
i_{c a} \\
i_{c b} \\
i_{c b} \\
V_{C 1} \\
V_{C 2}
\end{array}\right]= & {\left[\begin{array}{ccccc}
-\frac{r_{c}}{L_{c}} & 0 & 0 & \frac{1}{2 L_{c}} & -\frac{1}{2 L_{c}} \\
0 & -\frac{r_{c}}{L_{c}} & 0 & \frac{1}{2 L_{c}} & -\frac{1}{2 L_{c}} \\
0 & 0 & -\frac{r_{c}}{L_{c}} & \frac{1}{2 L_{c}} & -\frac{1}{2 L_{c}} \\
\frac{1}{2 C} & \frac{1}{2 C} & \frac{1}{2 C} & 0 & 0 \\
-\frac{1}{2 C} & -\frac{1}{2 C} & -\frac{1}{2 C} & 0 & 0
\end{array}\right]\left[\begin{array}{c}
i_{c a} \\
i_{c b} \\
i_{c b} \\
V_{C 1} \\
V_{C 2}
\end{array}\right] } \\
& +\left[\begin{array}{ccc}
\frac{V_{d c}}{2 L_{c}} & 0 & 0 \\
0 & \frac{V_{d c}}{2 L_{c}} & 0 \\
0 & 0 & \frac{V_{d c}}{2 L_{c}} \\
\frac{i_{c a}}{2 C} & \frac{i_{c b}}{2 C} & \frac{i_{c c}}{2 C} \\
\frac{i_{c a}}{2 C} & \frac{i_{c b}}{2 C} & \frac{i_{c c}}{2 C}
\end{array}\right]\left[\begin{array}{c}
u_{a} \\
u_{b} \\
u_{c}
\end{array}\right]+\left[\begin{array}{c}
\frac{e_{a}}{L_{c}} \\
\frac{e_{b}}{L_{c}} \\
\frac{e_{c}}{L_{c}} \\
0 \\
0
\end{array}\right] .
\end{aligned}
$$




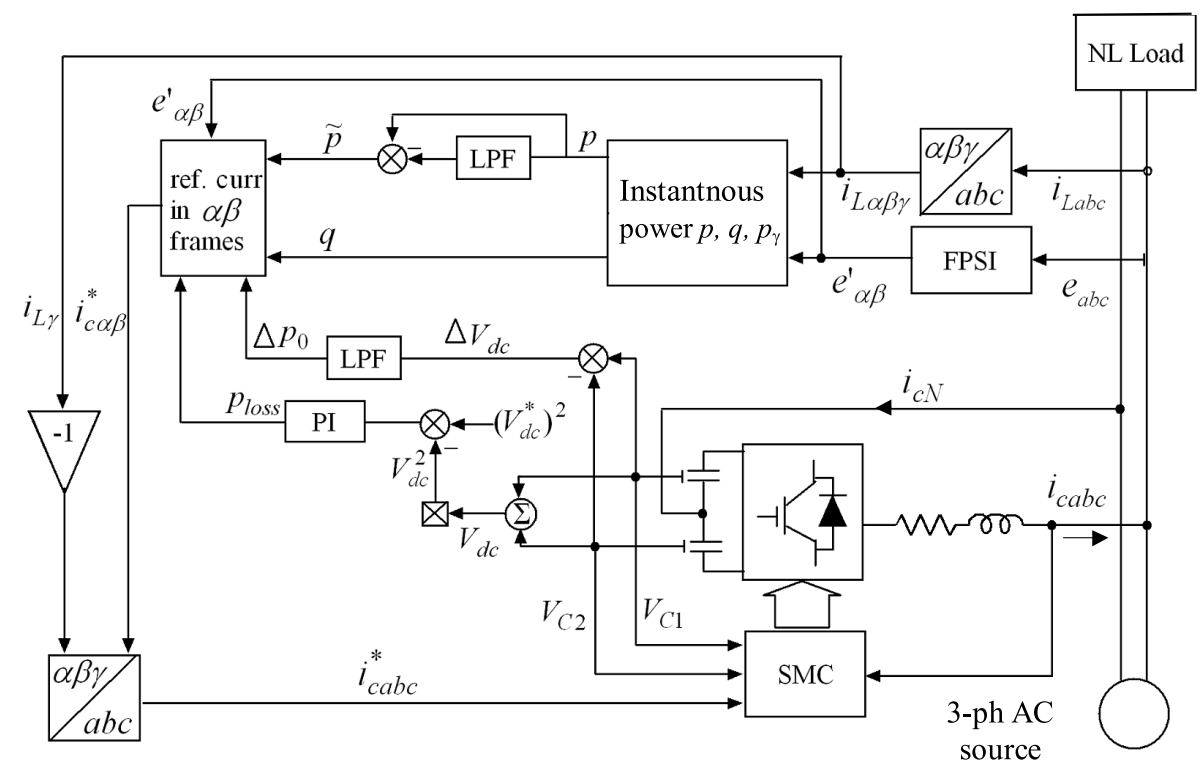

Fig. 3. Algorithm, used to optimize the power flow

\section{REFERENCE CURRENT IDENTIFICATION}

The reference current identification is needed to synthesize the component to be compensated from the measuring the load current and the voltage at the PCC. In this paper, the $p q$-theory method is adopted to achieve this operation. This technique proposed in [12] is expended and well explained in [3] for four-wire system applications. The principle of the $p q$-theory is to extract the compensated current components from the undesirable components in the instantaneous real, imaginary and zero-sequence powers. Consequently, if the mains voltages $e_{a b c}$ are disturbed, the instantaneous power will not reflect correctly the load current status, for this reason a Fundamental Positive Sequence Identification (FPSI) is generally included. Thus, if we denote $e_{\alpha}^{\prime}, e_{\beta}^{\prime}$, the instantaneous mains voltage corresponding to the correctly extracted fundamental positive sequence of $e_{a b c}$ in $\alpha \beta$ frames, then the instantaneous powers given in (11) will contain all spectrum information on load current.

$$
\left[\begin{array}{c}
p \\
q
\end{array}\right]=\left[\begin{array}{c}
\bar{p}+\tilde{p} \\
\bar{q}+\tilde{q}
\end{array}\right]=\left[\begin{array}{cc}
e_{\alpha}^{\prime} & e_{\beta}^{\prime} \\
-e_{\beta}^{\prime} & e_{\alpha}^{\prime}
\end{array}\right]
$$

Note that the new input voltage $e_{a b c}^{\prime}$ do not contain any zero-sequence voltage component $\left(e_{\gamma}=0\right)$, as a consequence, zero-sequence power $p_{\gamma}$ is also zero.

In ideal conditions, the optimal power flow is provided to the source when this one supplies only the average real power $\bar{p}$, this means that the active filter must compensate all other power components, and naturally the additional power components $p_{\text {loss }}$ and $\Delta p_{0}$ needed respectively to DC-bus voltage regulation and balancing, thus, the reference compensating powers are

$$
p_{c}^{*}=-\tilde{p}+p_{\text {loss }}+\Delta p_{0}, \quad q_{c}^{*}=-q .
$$

These signals are then used to calculate the following reference current in $\alpha \beta$-frames

$$
\left[\begin{array}{l}
i_{c \alpha}^{*} \\
i_{c \beta}^{*}
\end{array}\right]=\frac{1}{e_{\alpha}^{62}+e_{\beta}^{62}}\left[\begin{array}{cc}
e_{\alpha}^{\prime} & -e_{\beta}^{\prime} \\
e_{\beta}^{\prime} & e_{\alpha}^{\prime}
\end{array}\right]\left[\begin{array}{l}
p_{c}^{*} \\
q_{c}^{*}
\end{array}\right] .
$$

Since the zero-sequence current must be compensated, the reference component in the $\gamma$-axis is $i_{\gamma}$ itself with negative sign evidently. Finally, the reference currents in $a b c$-frames are obtained as follows

$$
\left[\begin{array}{c}
i_{c a}^{*} \\
i_{c b}^{*} \\
i_{c c}^{*}
\end{array}\right]=\sqrt{\frac{2}{3}}\left[\begin{array}{ccc}
\frac{1}{\sqrt{2}} & 1 & 0 \\
\frac{1}{\sqrt{2}} & -\frac{1}{2} & \frac{\sqrt{3}}{2} \\
\frac{1}{\sqrt{2}} & -\frac{1}{2} & -\frac{\sqrt{3}}{2}
\end{array}\right]\left[\begin{array}{c}
i_{L \gamma}^{*} \\
i_{c \alpha}^{*} \\
i_{c \beta}^{*}
\end{array}\right] .
$$

This algorithm is represented schematically in Fig. 3.

\section{SLIDING MODE CONTROL}

The sliding mode control consists to select the suitable switching configuration of the VSI in order to guarantee the state trajectory attraction toward a predefined sliding surface, and to maintain it stable over this surface.

The system established in (8) is a MIMO nonlinear system. In order to formulate the sliding mode creation problem, letting

$$
\mathbf{x}=\left[\begin{array}{lllll}
x_{1} & x_{2} & x_{3} & x_{4} & x_{5}
\end{array}\right]^{\top}=\left[\begin{array}{lllll}
i_{c a} & i_{c b} & i_{c c} & V_{c 1} & V_{C 2}
\end{array}\right]^{\top} .
$$

Then the state equation can be rearranged in the form

$$
\dot{\mathbf{x}}=\boldsymbol{f}(\mathbf{x})+\mathbf{G}(\mathbf{x}) \mathbf{u} .
$$

Where the $n$ dimensional vector field $\boldsymbol{f}(\mathbf{x})$, the $n \times m$ dimensional input matrix $\mathbf{G}(\mathbf{x})$ and the $m$ dimensional control vector $\boldsymbol{u}$ are given as follows

$$
\boldsymbol{f}(\mathbf{x})=\left[\begin{array}{c}
-\frac{r_{c}}{L_{c}} x_{1}+\frac{1}{2 L_{c}} x_{4}-\frac{1}{2 L_{c}} x_{5}-\frac{e_{a}}{L_{c}} \\
-\frac{r_{c}}{L_{c}} x_{2}+\frac{1}{2 L_{c}} x_{4}-\frac{1}{2 L_{c}} x_{5}-\frac{e_{b}}{L_{c}} \\
-\frac{r_{c}}{L_{c}} x_{3}+\frac{1}{2 L_{c}} x_{4}-\frac{1}{2 L_{c}} x_{5}-\frac{e_{c}}{L_{c}} \\
\frac{1}{2 C} x_{1}+\frac{1}{2 C} x_{2}+\frac{1}{2 C} x_{3} \\
-\frac{1}{2 C} x_{1}-\frac{1}{2 C} x_{2}-\frac{1}{2 C} x_{3}
\end{array}\right]=\left[\begin{array}{c}
f_{1}(\mathbf{x}) \\
f_{2}(\mathbf{x}) \\
f_{3}(\mathbf{x}) \\
f_{4}(\mathbf{x}) \\
f_{5}(\mathbf{x})
\end{array}\right],
$$




$$
\mathbf{G}(\mathbf{x})=\left[\begin{array}{ccc}
\frac{x_{4}+x_{5}}{2 L_{c}} & 0 & 0 \\
0 & \frac{x_{4}+x_{5}}{2 L_{c}} & 0 \\
0 & 0 & \frac{x_{4}+x_{5}}{2 L_{c}} \\
\frac{x_{1}}{2 C} & \frac{x_{2}}{2 C} & \frac{x_{3}}{2 C} \\
\frac{x_{1}}{2 C} & \frac{x_{2}}{2 C} & \frac{x_{3}}{2 C}
\end{array}\right]=\left[\begin{array}{l}
g_{1}(\mathbf{x}) \\
g_{2}(\mathbf{x}) \\
g_{3}(\mathbf{x}) \\
g_{4}(\mathbf{x}) \\
g_{5}(\mathbf{x})
\end{array}\right], \mathbf{u}=\left[\begin{array}{l}
u_{a} \\
u_{b} \\
u_{c}
\end{array}\right] .
$$

\subsection{Sliding surfaces}

For the $n$ dimensional controlled system regulated by $m$ independent switches, $m$ sliding surface coordinate functions are defined. The $m$ sliding surfaces are represented by the smooth algebraic restrictions $\sigma_{i}(\mathbf{x})=0$, $i=1,2, \ldots, m$. For each surface $S_{i}$, we have

$$
S_{i}=\left\{\mathbf{x} \in R^{n} \mid \sigma_{i}(\mathbf{x})=0\right\}
$$

And the intersection of the $m$ surfaces is denoted by $S$ which verify

$$
S=\left\{\mathbf{x} \in R^{n} \mid \mathbf{x} \in S_{i}, i=1,2, \ldots, m\right\} .
$$

Let define the vector of the sliding surface coordinate functions as follows

$$
\boldsymbol{\sigma}(\mathbf{x})=\left[\begin{array}{l}
\sigma_{1}(\mathbf{x}) \\
\sigma_{2}(\mathbf{x}) \\
\sigma_{3}(\mathbf{x})
\end{array}\right]=\left[\begin{array}{l}
x_{1}-x_{1}^{*} \\
x_{2}-x_{2}^{*} \\
x_{3}-x_{3}^{*}
\end{array}\right]
$$

We know yet that when the sliding mode is reached, in other words, when the state vector is forced to evolve on the intersection of the sliding surface, $i e \mathbf{x} \in S$, the sliding surface coordinate function $\boldsymbol{\sigma}(\mathbf{x})$ must satisfy the following condition

$$
(\dot{\boldsymbol{\sigma}}(\mathbf{x}), \boldsymbol{\sigma}(\mathbf{x}))=(\mathbf{0}, \mathbf{0})
$$

Then, we can write

$$
\dot{\boldsymbol{\sigma}}(\mathbf{x})=\frac{\partial \boldsymbol{\sigma}(\mathbf{x})}{\partial \mathbf{x}^{\top}}\left(\boldsymbol{f}(\mathbf{x})+\mathbf{G}(\mathbf{x}) \mathbf{u}_{e q}(\mathbf{x})\right)=\mathbf{0}
$$

We denote $\frac{\partial \boldsymbol{\sigma}(\mathbf{x})}{\partial \mathbf{x}^{\top}} \boldsymbol{f}(\mathbf{x})$ by $L_{f} \boldsymbol{\sigma}(\mathbf{x})$, an dimensional vector which represents the directional derivative of $\boldsymbol{\sigma}(\boldsymbol{x})$ along the direction of the vector field $\boldsymbol{f}(\mathbf{x})$. Similarly, the $m \times m$ dimensional matrix $\frac{\partial \boldsymbol{\sigma}(\mathbf{x})}{\partial \mathbf{x}^{\top}}$ is denoted by $L_{G}(\boldsymbol{\sigma}(\mathbf{x})$. Thus, (20) is rewritten as follows

$$
\dot{\boldsymbol{\sigma}}(\mathbf{x})=L_{f} \boldsymbol{\sigma}(\mathbf{x})+L_{G} \boldsymbol{\sigma}(\mathbf{x}) \boldsymbol{u}_{e q}(\mathbf{x})=\mathbf{0} .
$$

This permits to define the equivalent control in the form

$$
\mathbf{u}_{e q}(\mathbf{x})=-\left(L_{G} \boldsymbol{\sigma}(\mathbf{x})\right)^{-1} L_{f} \boldsymbol{\sigma}(\mathbf{x})
$$

This means that as a condition for the equivalent control definition is that the matrix $L_{G} \boldsymbol{\sigma}(\mathbf{x})$ must be invertible. Note also that the equivalent control must satisfy $\mathbf{- 1} \leq$ $\boldsymbol{u}_{e q}(\mathbf{x}) \leq \mathbf{1}$ which is the necessary and sufficient condition for the sliding mode existence over the surface $S$.
The expression of the equivalent control can be developed as follows

$$
\boldsymbol{u}_{e q}(\mathbf{x})=-\left(\frac{\partial \boldsymbol{\sigma}(\mathbf{x})}{\partial \mathbf{x}^{\top}} \mathbf{G}(\mathbf{x})\right)^{-1}\left(\frac{\partial \boldsymbol{\sigma}(\mathbf{x})}{\partial \mathbf{x}^{\top}} \boldsymbol{f}(\mathbf{x})\right)
$$

which permits to establish the equivalent control for the system as follows

$$
\mathbf{u}_{e q}(\mathbf{x})=\left[\begin{array}{l}
u_{e q a} \\
u_{e q b} \\
u_{e q c}
\end{array}\right]=\left[\begin{array}{l}
\frac{1}{x_{4}+x_{5}}\left(2 r_{c} x_{1}-x_{4}+x_{5}+2 e_{a}\right) \\
\frac{1}{x_{4}+x_{5}}\left(2 r_{c} x_{2}-x_{4}+x_{5}+2 e_{b}\right) \\
\frac{1}{x_{4}+x_{5}}\left(2 r_{c} x_{3}-x_{4}+x_{5}+2 e_{c}\right)
\end{array}\right] .
$$

Since the sliding mode is reached the state equation of the system is expressed as

$$
\dot{\boldsymbol{x}}=\boldsymbol{f}(\mathbf{x})-\mathbf{G}(\mathbf{x})\left(L_{G} \boldsymbol{\sigma}(\mathbf{x})\right)^{-1} L_{f} \boldsymbol{\sigma}(\mathbf{x}) .
$$

\subsection{Sliding surface accessibility}

Let consider the following Lyapunov function

$$
V(\boldsymbol{\sigma}(\mathbf{x}))=\frac{1}{2} \boldsymbol{\sigma}^{\top}(\mathbf{x}) \boldsymbol{\sigma}(\mathbf{x}) .
$$

It is a semi-definite function, it is identically zero over the surface $S$, ie when $\boldsymbol{\sigma}(\mathbf{x})=\mathbf{0}$ and positive when $\boldsymbol{\sigma}(\mathbf{x}) \neq \mathbf{0}$. The quantity $V(\boldsymbol{\sigma}(\mathbf{x}))$ can be interpreted as the distance from the position of the point $\mathbf{x}$ in the state space to the desired surface $S$. Therefore, in order to satisfy the condition $\boldsymbol{\sigma}(\mathbf{x}) \neq \mathbf{0}$, the discrete control $\mathbf{u}$ must exercise a closing or opening action, which permits to decrease the distance $V(\boldsymbol{\sigma}(\mathbf{x}))$, this means that the variation of this function in the time must be strictly negative, then

$$
\frac{\mathrm{d}}{\mathrm{d} t} V(\boldsymbol{\sigma}(\mathbf{x}))=\boldsymbol{\sigma}^{\top}(\mathbf{x}) \dot{\boldsymbol{\sigma}}(\mathbf{x})<0 .
$$

This is the condition for the trajectory attraction toward the sliding surface.

Referring to (21) and (27), if $\boldsymbol{\sigma}(\mathbf{x}) \neq \mathbf{0}$, replacing $\mathbf{u}_{e q}(\mathbf{x})$ by $\mathbf{u}$, then the time derivative of the Lyapunov function can be expressed as follows

$$
\dot{V}(\boldsymbol{\sigma}(\mathbf{x}))=\boldsymbol{\sigma}^{\top}(\mathbf{x})\left(L_{f} \boldsymbol{\sigma}(\mathbf{x})+L_{G} \boldsymbol{\sigma}(\mathbf{x}) \mathbf{u}\right)<0 .
$$

Likewise, if $\boldsymbol{\sigma}(\mathbf{x})=\mathbf{0}$, then

$$
\dot{V}(\boldsymbol{\sigma}(\mathbf{x}))=\boldsymbol{\sigma}^{\top}(\mathbf{x})\left(L_{f} \boldsymbol{\sigma}(\mathbf{x})+L_{G} \boldsymbol{\sigma}(\mathbf{x}) \mathbf{u}_{e q}\right)=0 .
$$

Now, if we consider that the switching frequency is infinite or sufficiently high, we can suppose with good approximation that the state vector $\mathbf{x}$ takes the same value in the both case (28) and (29). Thus, subtracting (29) from (28), the restriction (27) can be rewritten as follows

$$
\dot{V}(\boldsymbol{\sigma}(\mathbf{x}))=\boldsymbol{\sigma}^{\top}(\mathbf{x}) L_{G} \boldsymbol{\sigma}(\mathbf{x})\left(\mathbf{u}-\mathbf{u}_{e q}(\mathbf{x})\right)<0 .
$$

This inequality can be achieved by applying the control vector given by

$$
\mathbf{u}=-\operatorname{sign}\left(\boldsymbol{\sigma}^{\top}(\mathbf{x}) L_{G} \boldsymbol{\sigma}(\mathbf{x})\right)^{\top} .
$$

And finally, the switch position functions

$$
\boldsymbol{d}=\frac{1}{2}\left(\mathbf{1}-\operatorname{sign}\left(\boldsymbol{\sigma}^{\top}(\mathbf{x}) L_{G} \boldsymbol{\sigma}(\mathbf{x})\right)^{\top}\right) .
$$

Where sign designs the sign function and $\mathbf{1}$ is an $m$ dimensional column vector constituted of 1 in each entry. 

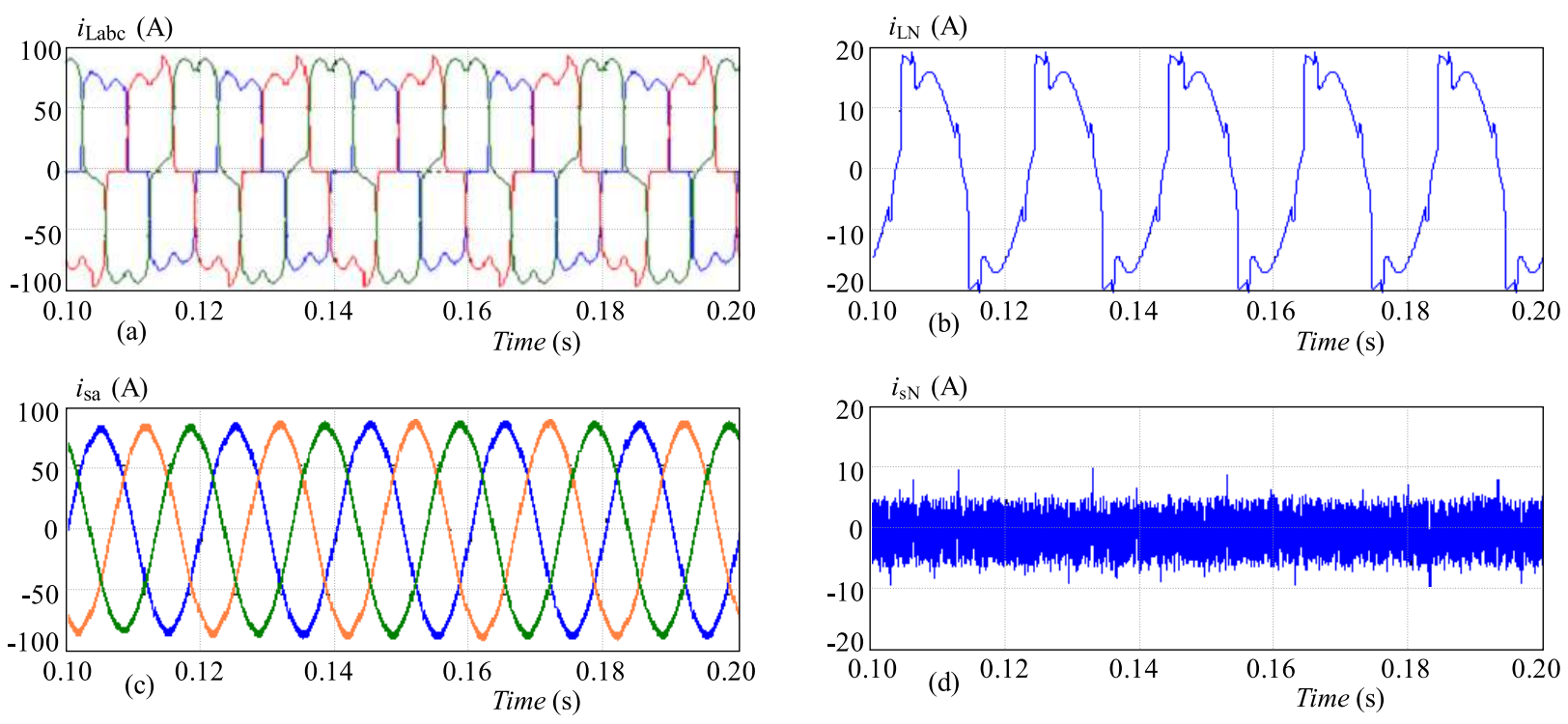

Fig. 4. The compensation of three-phase and the neutral currents
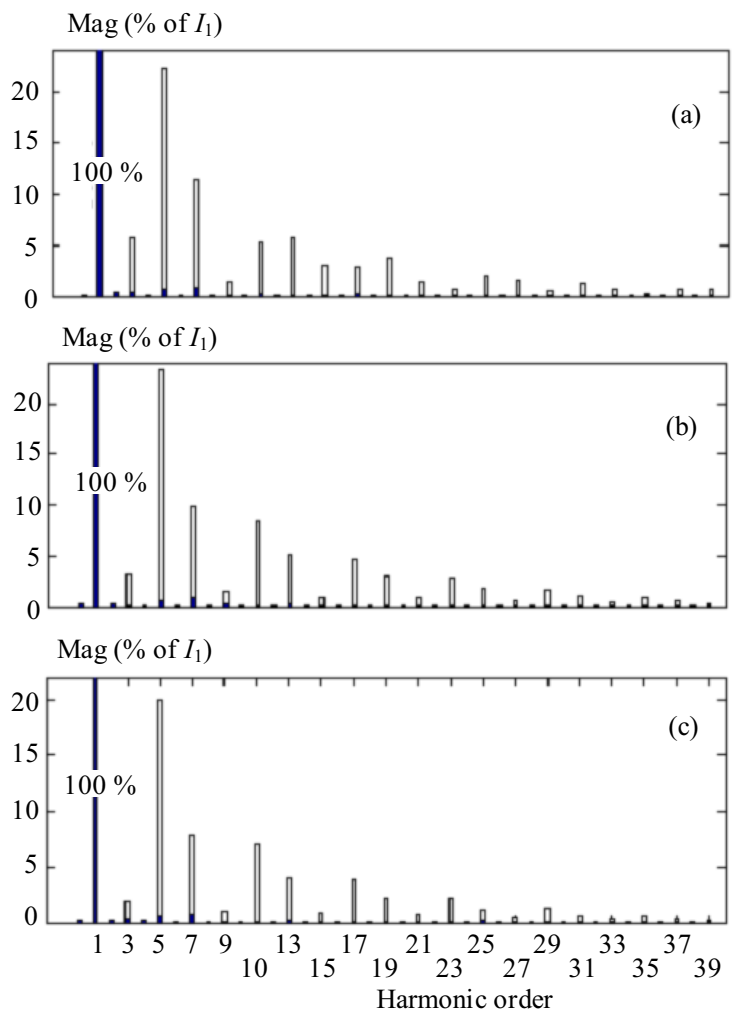

Fig. 5. Harmonics spectrum of the mains currents before and after compensation
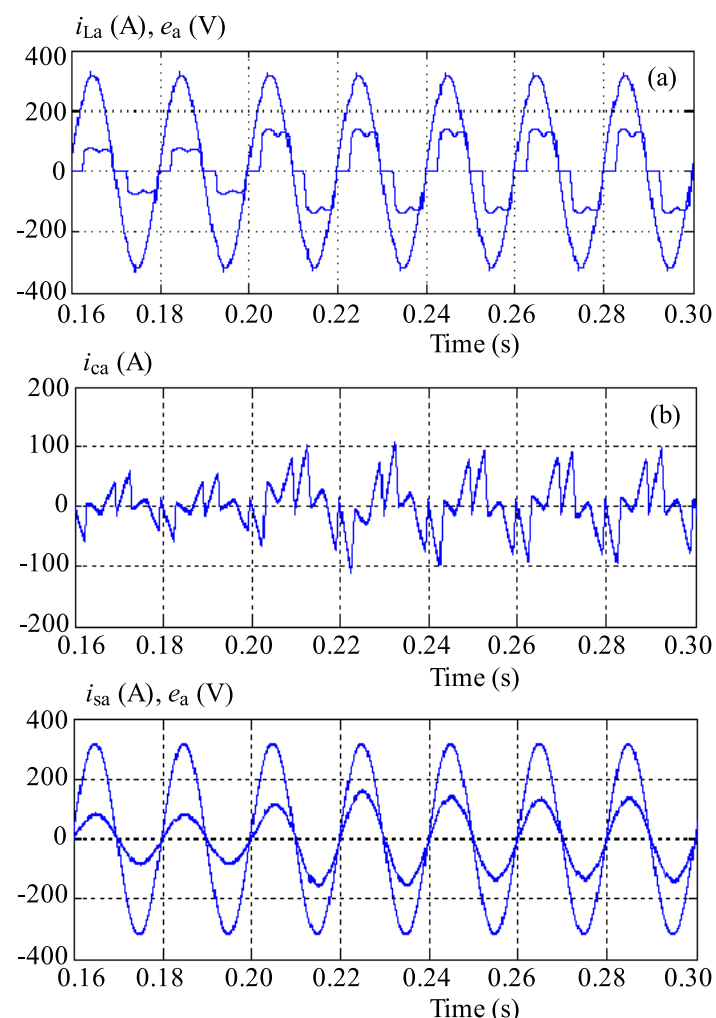

Fig. 6. Harmonics and reactive power compensation under load change

\section{SIMULATION RESULTS}

The performances of the developed sliding mode control were verified through simulation using MATLAB software. The polluting load is constituted with threephase thyristor rectifier, single-phase thyristor rectifier and single-phase diode rectifier. The resulting load currents are thus deformed and unbalanced. The mains parameters of the system are

Phase-to-neutral voltage source $230 \mathrm{~V} \mathrm{rms}, 50 \mathrm{~Hz}$

$$
\begin{aligned}
& L_{s}=100 \mu \mathrm{H} \\
& C_{1}=C_{2}=5 \mathrm{mF} \\
& V_{d c}^{*}=1000 \mathrm{~V} \\
& L_{c}=2 \mathrm{mH}
\end{aligned}
$$

The mains simulation results are illustrated in the following figures. First, in Fig. 4 it can be seen that the three-phase and the neutral currents are correctly compensated. In fact, the spectrum analysis in Fig. 5 shows that without active filtering, the mains current contain 

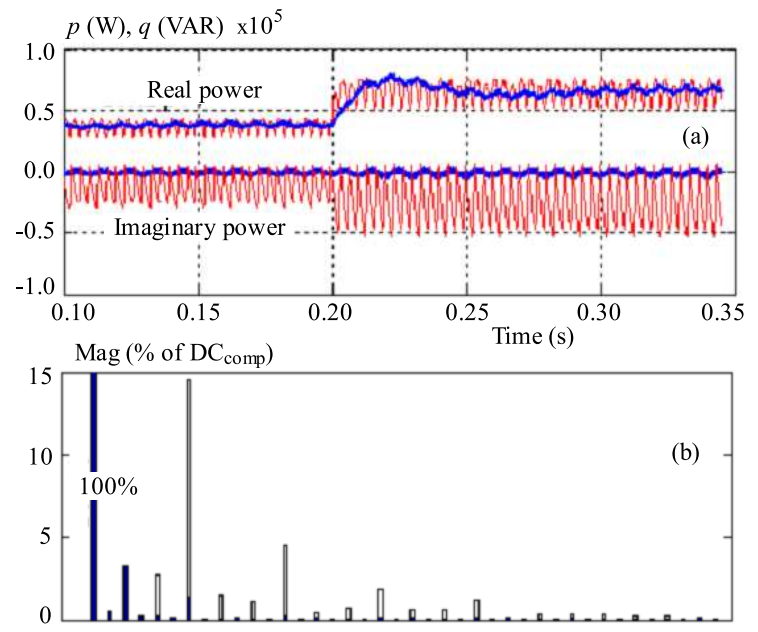

$\operatorname{Mag}\left(\%\right.$ of $\left.\mathrm{DC}_{\text {comp }}\right)$

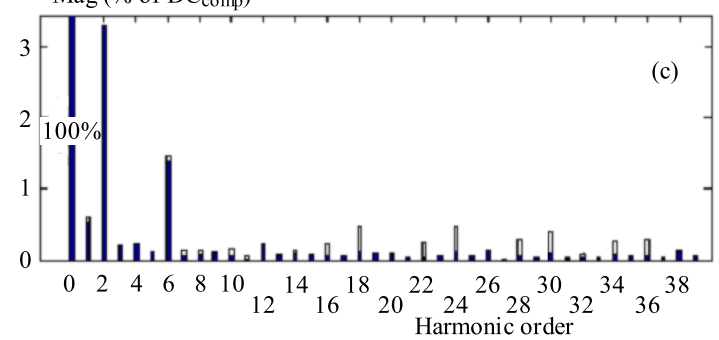

Fig. 7. The instantaneous, real and imaginary power
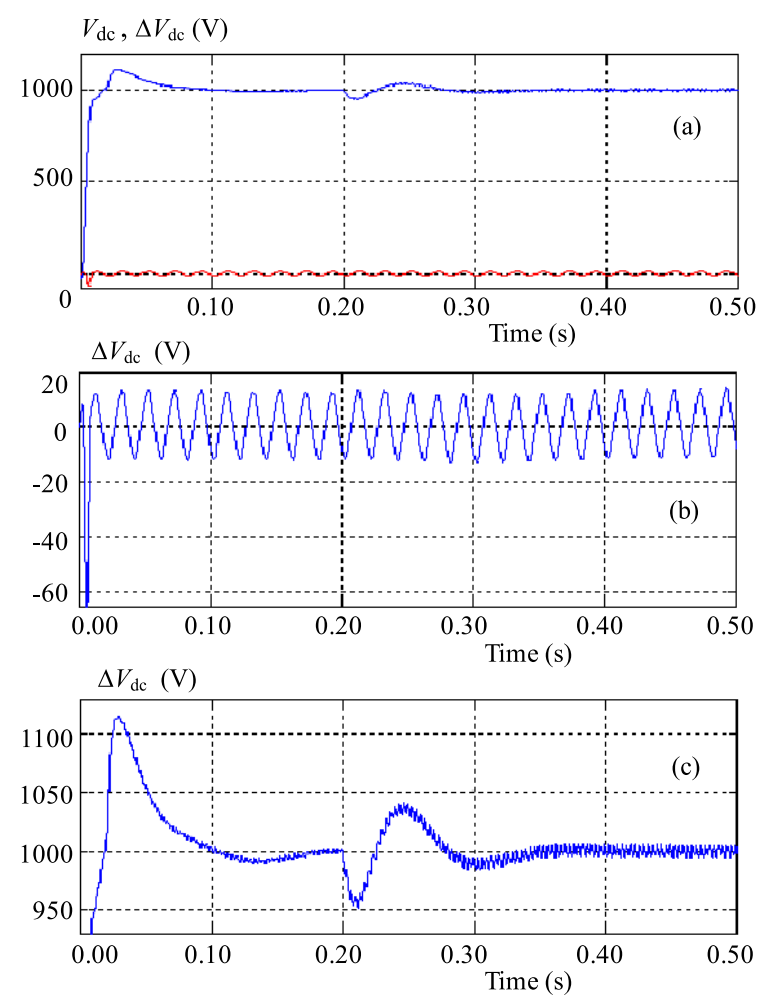

Fig. 8. The DC-bus voltage regulation

positive sequence harmonics $6 h+1\left(7^{\text {th }}, 13^{\text {th }}, \ldots\right)$, negative sequence harmonics $6 h-1\left(5^{\text {th }}, 11^{\text {th }}, \ldots\right)$, and zero sequence harmonics $6 h-3\left(3^{\text {rd }}, 9^{\text {th }}, \ldots\right)$, with $h \geq 1$ designs the harmonic order. Note that the zero sequence harmonics is the result of the $4^{\text {th }}$-wire (neutral), this se- quence does not appears in the three-wire systems. With introducing active filtering action, these components are almost canceled.

Figure 6 illustrates the performances of the active filter controller to guarantee source current in sinusoidal form and in phase with mains voltage, under load change, where it can be seen that the power factor and the total harmonic distortion are both excellently improved before and after load change. This can be observed also in Fig. 7 where the instantaneous real and imaginary power is represented. The imaginary power is almost canceled, and the real power drawn from the $\mathrm{AC}$ source is practically free of the alternating part of the load power. The real power spectrum in Fig. 7(b) shows effectively that the $2 h^{\text {th }}$ harmonic order components in the load power are mitigated in the source side. This quality is also observed after load change as it is illustrated in Fig. 7(c).

Table 1. Detailed results of three-phase and neutral currents compensation

\begin{tabular}{lcccc}
\hline \multirow{2}{*}{-phase, Neutral } & \multicolumn{2}{c}{$t<0.2 \mathrm{~s}$} & \multicolumn{2}{c}{$t>0.2 \mathrm{~s}$} \\
& Load & Source & Load & Source \\
\hline THD (\%) & & & & \\
$a$ phase & 28.29 & 01.60 & 26.15 & 02.00 \\
$b$ phase & 27.67 & 01.45 & 24.83 & 01.86 \\
$c$ phase & 23.76 & 01.76 & 23.59 & 01.90 \\
Neutral & 31.31 & - & 31.31 & - \\
RMS & & & & \\
$a$ phase & 54.03 & 54.34 & 98.42 & 93.12 \\
$b$ phase & 58.74 & 54.73 & 105.57 & 93.05 \\
$c$ phase & 65.21 & 54.44 & 108.57 & 92.48 \\
Neutral & 13.10 & - & 13.10 & - \\
\hline
\end{tabular}

Table 2. Detailed results of instantaneous real and imaginary powers compensation

\begin{tabular}{lcccc}
\hline 3-phase, Neutral & \multicolumn{2}{c}{$t<0.2 \mathrm{~s}$} & \multicolumn{2}{c}{$t>0.2 \mathrm{~s}$} \\
& Load & Source & Load & Source \\
\hline THD (\%) & & & & \\
$p$ & 15.98 & 03.66 & 16.85 & 03.69 \\
$q$ & 107.6 & - & 87.90 & - \\
DC component & & & & \\
$\bar{p}\left(\times 10^{4}\right)$ & 03.80 & 03.71 & 06.64 & 06.56 \\
$\bar{q}\left(\times 10^{4}\right)$ & 01.29 & - & 02.58 & - \\
\hline
\end{tabular}

Tables 1 and 2 summarize the detailed results of current and instantaneous power compensation. Note that the total harmonic distortion of the real power is computed to DC component.

The DC-bus voltage regulation is shown in Fig. 8 the DC-bus is charged and the voltage level $V_{d c}$ reaches the predefined value $V_{d c}^{*}$ with excellent response $(950 \mathrm{~V}<$ $V_{d c}<1050 \mathrm{~V}$ in $0.05 \mathrm{~s}$ ). The excellent dynamic performance is also observed with load change operated at $t=0.2 \mathrm{~s}$, in fact the DC-link voltage is remained inside 
the bounded range $[950 \mathrm{~V}, 1050 \mathrm{~V}]$ and stabilized at $V_{d c}^{*}$ in $0.1 \mathrm{~s}$. The DC-link unbalance is shown in Fig. 8(c) where one can remark that the two voltage $V_{C 1}$ and $V_{C 2}$ are sufficiently balanced, accept the existence of small oscillation $\Delta V_{d c}$ (about $1.3 \%$ of $V_{d c}$ ) which can be neglected. It should be noted that the DC-link voltage oscillation $V_{d c}$ and $\Delta V_{d c}$ are strongly depending of the capacitors value. In fact, it is known that to keep these oscillation inside an acceptable bounded ranges, generally a large capacitors is recommended, the two capacitors increase considerably when $C_{1}$ and $C_{2}$ are chosen small, for this reason, in this paper this parameters is relatively large.

\section{CONCLUSION}

In this paper, a sliding mode control for three-phase three-leg voltage source inverter based four-wire shunt active filter is applied. The system is observed as a MEMOdecoupled nonlinear system, which permits a simple implementation. The simulation results show the ability of this method to track references with minimum error, fast response and high robustness. A low line current THD, and high power factor are provided even load change.

Finally, as critics, it should be noted that, the decoupled nature of the used VSI topology is simple to analyze but we have seen that it need very large capacitors value in the DC-bus.

For the sliding mode control, it is known that the switching frequency presents its principal incontinent. The replacement of the three-leg topology by the four-leg one can resolve the problem of first mentioned critic, and we know yet that the switching frequency stabilization is possible. This will make the object of the continuation for this work.

\section{REFERENCES}

[1] AKAGI, H.: New Trends in Active Filter for Power Conditioning, IEEE Transactions on Industry Applications, 32 No. 6 (Dec 1996), 1312-1322.

[2] Al-HADDAD, S. K.-CHANDRA, A.: A Review of Active Filters for Power Quality Improvement, IEEE Transaction on industrial Electronics 46 No. 5 (Oct 1999), 960-971.

[3] AREDES, M.-HÄFNER, J.-HEULMANN, K. : Three-Phase Four-Wire Shunt Active Filter Control Strategies, IEEE Transaction on Power Electronics 12 No. 2 (March 1997), 311-318.

[4] SINGH, B.-Al-HADDAD, K.-CHANDRA, A.: Harmonic Elimination, Reactive Power Compensation and Load Balancing in Three-Phase, Four-Wire Electric Distribution Systems Supplying Non-Linear Loads, Electric Power Systems Research 44 (1998), 93-100.

[5] UCAR, M.-OZDEMIR, E. : Control of a 3-Phase 4-Leg Active Power Filter under Non-Ideal Mains Voltage Condition, Electric Power Systems Research (2007).
[6] UTKIN, V. A. : Variable Structure Systems with Sliding Mode, IEEE Transaction on Automatic Conbtrol AC22 No. 2 (1997), 1105-1120.

[7] SPIAZZI, G.-MATTAVELLI, P.—ROSSETTO, L.-ALESANI, L.: Application of Sliding Mode Control to Switch-Mode Power Supplies, Journal of Circuits, Systems and Computers (JCSC) 5 No. 3 (Sep 1995), 337-354.

[8] AHMED, M.: Sliding Mode Control for Switched Mode Power Supplies, PhD thesis, Lappeenranta University of Technology, Finland, 2004.

[9] SIRA-RAmiREZ, S.-SILVA-ORTIGOZA, R. : Control Design Techniques in Power Electronics Devices, Springer-Verlag, London, 2006.

[10] SABANOVIC, N.-NINOMIYA, T.-SABANOVIC, A. PERUNICIC, B.: Control of Three-Phase Switching Converters: A Sliding Mode Approach, PESC'93, 1993, pp. 630-635.

[11] MENDALEK, N.-Al-HADDAD, K.-FNAIECH, F.-DESSAINT, A.: Sliding Mode Control of 3- Phase 3- Wire Shunt Active Filter in the $d q$ Frame, IEEE CCECE'2001, 2001, pp. $765-770$.

[12] AKAGI, H.-KANAZAWA, Y.-NABAE, A.: Generalized Theory of the Instantaneous Reactive Power in Three-Phase Circuits, in IPEC'83Int. Power Elec. Conf., Tokyo, Japan, 1983, pp. $1375-1386$

Received 18 October 2009

Farid HARMOUDI was born in Bejaia, Algeria in 1980. He received the engineer diploma from the University of Bejaia, and the M.S degrees from the University of Batna in electrical engineering in 2005 and 2008 respectively. He is actually working toward the PHD thesis. His area of research includes power electronics, harmonics in power systems and active harmonics filtering. From December 2009, he is an assistant teacher at the Institute of Electrical Engineering and Electronics, at the University of Boumerdes, Algeria.

Abdelaziz CHAGHI was born in Batna, Algeria, 1954. He received his BS degree from the University of Oran, Algeria 1980, and Master degree from the University of Manchester, England 1984, and received his PhD from Batna University, Algeria 2004. He is currently a Professor at department of Faculty of Technology, Electrical Engineering at Batna University. His areas of interest include power systems optimization, power system protection and power quality

Mouloud ADLI was born in Tizi Ouzou, Algeria in 1957. He received the engineer diploma in electrical engineering from the Ecole Nationale Polytechnique, Algiers, Algeria in 1984 and the Doct. Ing diploma from the T.U. University of Magdeburg, Germany in 1993. He is now working as assistant professor and a researcher in the Electrical Engineering Research Laboratory at the University of Bejaia. His areas of interest include fuzzy control, harmonics in power systems and power electronics.

Hocine AMIMEUR was born in Bejaia, Algeria in 1977. He received the engineer diploma from the University of Bejaia, and the M.S degrees from the University of Batna in electrical engineering in 2005 and 2008 respectively. He is actually working toward the PHD thesis. His area of research includes power electronics, Induction machines and renewable energies. From February 2011, he is an assistant teacher at the Electrical Engineering Department, at the University of Bejaia, Algeria 